\title{
Reckoning physical properties of firewood with its preference by the rural households in a selected village of Narsingdi district of Bangladesh
}

\author{
Md. Danesh Miah ${ }^{(1,2)^{*}}$, Gazi Azizul Islam ${ }^{(1)}$ \\ ${ }^{(1)}$ Institute of Forestry and Environmental Sciences, University of Chittagong, Chittagong, Bangladesh \\ ${ }^{(2)}$ Forest Policy Laboratory, Faculty of Agriculture, Shinshu University, Nagano, Japan
}

Received: 20 December 2018

Accepted: 21 November 2020

Published online: 31 December 2020

\begin{abstract}
The biomass energy has the potentiality of sequestering carbon and the capacity of reducing global warming. The study was conducted with a view to assessing the local preferences of firewood for domestic use with the physical characteristics of firewood species. The study was conducted in different communities of Kamrabo village under Shibpur Upazila in Narsingdi district of Bangladesh. Purposive random sampling was performed for the study covering 50\% households with a total of 160 households of the study area. A direct interview with semi-structured questionnaires based on twelve quality criteria was conducted in the study area for identification of local preferences of firewood species and drawing up a pair-wise ranking matrix based on it. Fuel value index (FVI) was used to rank the preferred firewood species based on their physical properties. The trees Artocarpus heterophyllus, Swietenia mahagoni, Albizia lebbek, Acacia auriculiformis, Syzygium fruticosum, etc. having the FVI values were found in decreasing order. The study found a resemblance between the order of FVI values and the ranks of the firewood species by household preferences. The study will be useful in energy management and policy in rural Bangladesh.
\end{abstract}

Keywords: firewood preferences; fast drying; easy flammability; hot flame; physical properties of firewood

\section{Introduction}

Biomass produces more energy than other forms of energy and its world consumption is eight times greater than the total of all other energy sources and presently its contribution is about $10-14 \%$ of the world's energy supply (Balat and Ayar 2005; McKendry 2002). It has the capacity of reducing the global warming as renewable energy sources being a component of the carbon neutral systems and substituting fossil fuels for heat and electricity generation (Akther et al. 2010c; Werther et al. 2000). Forest biomass also attracts global and local attention for mitigation of global climate change by reducing greenhouse gases and meeting increased energy demand having easy conversion process into secondary energy carriers with a lower capital investment (Miah et al. 2011).

\footnotetext{
* Corresponding author: Md. Danesh Miah

e-mail:dansmiah@gmail.com; danesh@cu.ac.bd
} 
In the rural area, biomass is an important alternative for heat and energy supply and short rotation plantation meets the demand of woodfuel (Bungart and Huttl 2001). In rural Bangladesh, biomass fuel meets about $76 \%$ of total fuel where about $74 \%$ of biomass fuels are collected from the own homestead and agricultural land (Akther et al. 2010a; Eusuf 1997). Rational and responsible use of biomass reduces the rural poverty as it is locally available, having no dependency on the price fluctuations and supply uncertainties (Demirbas 2004; McKendry 2002; Singh et al. 2000). Among different energy sources (LPG, kerosene, biomass, electricity, and candle), about $92 \%$ households use biomass in disregarded villages of Bangladesh and, wood and agricultural residues represent $40 \%$ of total energy consumption which is worth about US\$ 7 billion per year in ASEAN countries (FAO 1997; Miah et al. 2010). The main biomass energy sources are wood, bamboo, branches, cow dung, firewood, rice husk, leaves and twigs, shell, and coir of coconut, sawdust, straw agricultural crops and wastes, fast-growing trees and plants, aquatic plants, animal wastes, livestock operation residues, municipal and industrial wastes etc. (Akther et al. 2010b; Huda et al. 2014; Jashimuddin et al. 2006; Lucia et al. 2006; Miah et al. 2010). The biomass energy is mainly used in domestic cooking, space heating, tea stalls, brickfields, bakeries, paddy parboiling, smithies and potteries (FAO 1997; Jashimuddin et al. 2006; MacDonald et al. 2001).

The fuelwood qualities have an association with the preferences of the local population. The socially defined properties of firewood are flammability, flame brightness, flaming period, odor, smoke released during the burning of wood, ease of ignition and ease of collection (Abbot and Lowore 1999; Cuvilas et al. 2014; Ramos et al. 2008). The local preference also depends on physical properties of plants indicating the highest Fuel Value Index (FVI) of plants in the specific region of countries (Ramos et al. 2008). The physical properties of firewood are calorific value, ash content, density and moisture content (Bhatt and Todaria 1990; Jain and Sing 1999; Kataki and Konwer 2002; Puri et al. 1994; Purohit and Nautiyal 1987).

In the physical properties, density and moisture content are important criteria for assessing physical characteristics of firewood, because high density indicates the high energy content per unit volume, burning for longer periods of time and better quality embers and on the other hand, low moisture content denotes the slow burning rate of wood, decreasing its calorific value, improvement of flammability and reduction of the weight of air-dry wood (Abbot et al. 1997; Abbot and Lowore 1999; Fuwape and Akindele 1997; Kataki and Konwer 2002; Mitchual et al. 2014). The high calorific values and low ash content of wood are considered for good fuelwood species (Bhatt et al. 2010). The high ash content is less desirable because it affects the calorific value of wood as it is dominated by calcium, silicon, aluminum, potassium, and magnesium (Kataki and Konwer 2002).

Energy consumption by households is influenced by several socioeconomic factors such as family size, income, household land ownership, educational level, etc. (Pachauri 2004; Rao and Reddy 2007). Several studies on energy allocation for cooking, rural household energy consumption, energy efficiency, and its utilization were reported for India, China, Sri Lanka, and Bangladesh, respectively (Agrawal and Singh 2001; Jashimuddin et al. 2006; Miah et al. 2003; Wijayatunga and Attalage 2002; Xiaohua and Zhenming 1996). Studies on homestead biomass energy situation and its utilization in the rural area of Bangladesh were reported by some authors e.g. Akhter et al. (1999), Miah et al. (2003) and Sarker and Islam (1998). Several studies were conducted in Bangladesh showing the household biomass fuel energy situation in poor forest and forest rich regions covering fuel use, fuel preference and consumption pattern in rural area (Akhter et al. 1999; Jashimuddin et al. 2006; Miah et al. 2003; Sarker and Islam 1998). There is a significant relationship between physical properties and local preferences of firewood (Abbot et al. 1997; Deka et al. 2007; Ramos et al. 2008). High-density species can produce better ember (Deka et al. 2007) and high moisture content species were prone to decay and insect attack (Purkayastha 1989). Low ash content enhances the FVI values (Abbot et al. 1997; Bhatt et al. 2010), but several studies on firewood species was conducted in Bangladesh which identified 
few social factors such as availability of fuelwood in home garden, easy propagation, low cost, high calorific value and easiness of handling, etc. for choosing firewood (Jashimuddin et al. 2006; Mukul et al. 2014). However, there was no significant study on showing the relationship between social preference factors and the FVI.

In spite of possibilities of mitigating the climate change and meeting increased demand of energy in Bangladesh, the knowledge pool lacks understanding the physical properties of firewood with its preferences by the rural households to encourage local people to plant high energy efficient tree species. So, this study was conducted with the objectives of assessing physical properties of firewood with its local preferences in Narsingdi district of Bangladesh. This study will be helpful to take necessary measures to proper utilization of biomass fuels by rural household in Bangladesh.

\section{Data sources and methods}

\section{Selection of study area and tree species}

Kamrabo village under Shibpur Upazila in Narsingdi district of Bangladesh was selected purposively for a study area (Fig. 1). It lies between $24^{\circ} 04^{\prime}$ and $23^{\circ} 06^{\prime} \mathrm{N}$ latitudes and $90^{\circ} 47^{\prime}$ and $90^{\circ} 49^{\prime}$ E longitudes. The study was conducted from November 2015 to January 2016 for the information of 12 quality criteria of fire-wood species.

The climatic condition of the study area is relatively mild both in the summer and the winter. During the winter, temperature varies from $19^{\circ} \mathrm{C}$ to $23.7^{\circ} \mathrm{C}$ and in summer, it varies from $26^{\circ} \mathrm{C}$ to $29^{\circ} \mathrm{C}$. The annual average rainfall of this area is $2376 \mathrm{~mm}$ and the soil of the study area is formed by grey silty complex clay for the young Brahmaputra floodplain. The areas under the Narsingdi district are also occupied by compacted, pale brown, sandy soils on the ridges and shallow, dark grey, compact clays overlying sand in the shallow basins. Topsoils are acidic in nature when dry but near neutral when flooded. Sub-soils are often slightly acidic at the top but all become moderately alkaline below (BBS 2013). The village is plentiful of the homestead and natural forest. The local people of Narsindi district largely depend on firewood for meeting their energy demand. The dominant species in the area are Jackfruit (Artocarpus heterophyllus), Mehegoni (Swietenia mahagoni) and Mango (Mangifera indica) (BBS 2013; Miah and Hossain 2002).

A socioeconomic survey was conducted in the study area covering the $50 \%$ household to find out the preference of firewood. Branches of thirty-six distinct trees of most preferred firewood species were selected for the analysis of physical characteristics in the laboratory.

\section{Selection of the respondents}

The study was conducted among five communities (Para) in Kamrabo village under Shibpur Upazila in Narsingdi district which were Pal para, Jhulhartek, Gazi bari, Bhuiya bari, and Saiyod para. There were 160 households in the village. Fifty percent (80) of this total household were selected randomly. A direct interview with the semi-structured questionnaire was conducted in households. Head of the household was the respondent of the study. Among the respondents, the study found that about $58 \%$ were female and $43 \%$ were male. Although the households in the study area are generally maledominated, a recent employment trend has changed the structure of the household. Presently, most of the male household heads live abroad for their jobs. Hence, the respective housewives play a vital role in the maintenance of household activities. They work as operational head of the households. So, most of the respondents were found female.

In the survey, there were respondents of different ages. The highest numbers of respondents (34\%) were in age between 30 to 40 years and only about 3\% respondents were in age between 60 to 
70 years. At present most of the people of Bangladesh are middle-aged and they have the ability to contribute for economic development of the country.

In the study, there were varieties of occupation. The occupations of respondents were the housewife, farmer, local businessmen, service holder, expatriate, and others. The highest numbers of respondents $(58 \%)$ were the housewife and only about $3 \%$ respondents were expatriate.

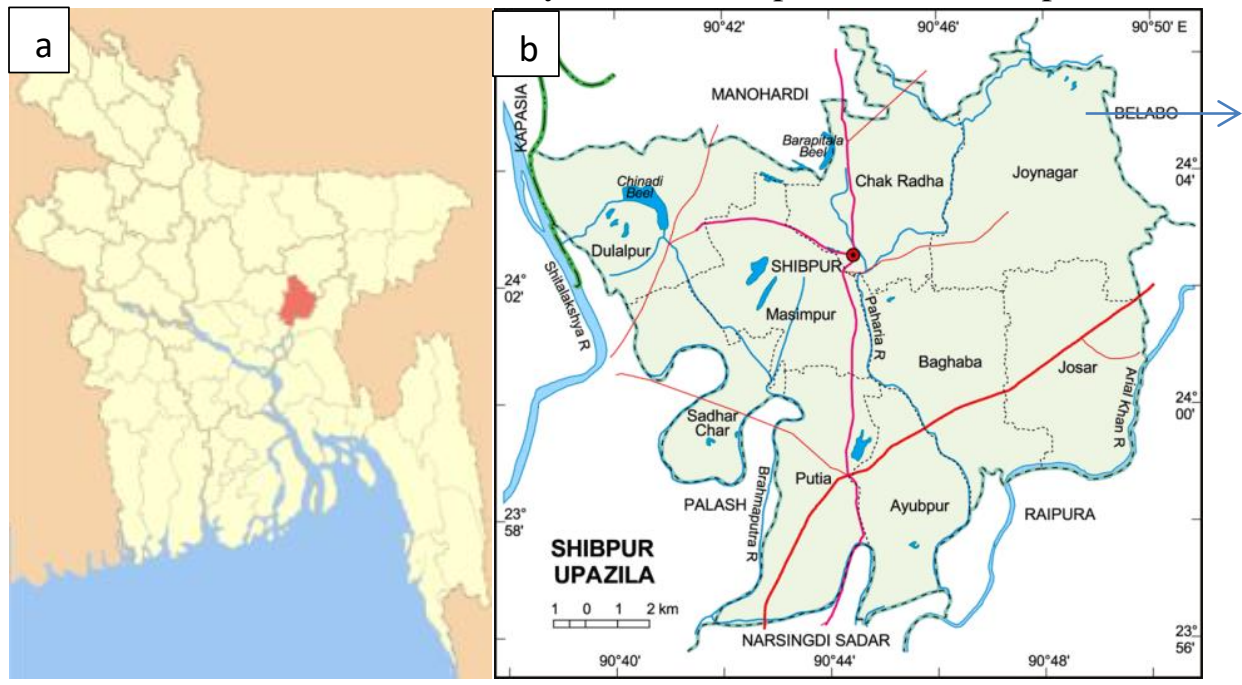

Fig. 1. Map of Bangladesh (a) and the map of study area, Kamrabo village under Shibpur Upazilla in Narsingdi district (b), Bangladesh.

\section{The present condition of the biomass fuel use}

In the study, about $51 \%$ household relied on their own land for firewood collection. On the other hand, about $20 \%, 15 \%, 6 \%, 4 \%$, and $3 \%$ households depend on own land and neighbor land, local market and own land, neighbor land, other land, and local market, respectively. Only $1 \%$ household depends on the local market and neighboring land for meeting their firewood demand. Local people collect firewood from their own land generally homestead forest. Miah et al. (2003) reported that the rich household obtained $44 \%$ firewood from homestead while poor household collected $74 \%$ firewood from secondary forests. Akther et al. (2010c) also reported that 56\% households collected biomass from their own land while $18 \%$ households collected from their neighbors' land.

Local people use different parts of firewood for cooking purpose. In the survey, it was found that the highest numbers of households (63\%) used branches as firewood while about only 5\% household used the trunk as firewood for cooking. The study in the rural area in Noakhali district of Bangladesh showed that about $70 \%$ of rural people used branches as fuel from their own homestead and agricultural land (Chowdhury et al. 2011). Akther et al. (2010c) also reported that $63 \%$ rural households used branches as firewood and highest portion of biomass energy as branches (86\%) used for cooking purposes in the rural area of Bangladesh.

\section{Data Analysis}

In the study, a pair-wise ranking was used to identify firewood properties and to rank the tree species. It was done using 12 quality criteria of fire-wood species to characterize the overall quality of the fuel. A matrix was drawn up comparing species and properties and the score for each species and criteria. Each quality criterion was made score ranging from 0 to 10. Rank was made on the basis of an obtained score and its range was 1 (best) to 10 (worst). The top preferred firewood species were selected on the basis of provided score. The selected tree species were identified for sampling. Samples of randomly selected branch cutting (diameter between 1.5 to $4 \mathrm{~cm}$ ) of selected tree species were harvested 
from the study area and each of them having 3 replications with the length of $10 \mathrm{~cm}$ were undertaken. Samples were packed carefully in polythene bags to transfer in the lab (Bhatt et al. 2010).

Thirty-six preferred or commonly used firewood species were tested in the wood analysis. Ranking of firewood species, an alternative ordering to the pair-wise ranking procedure was performed using FVI (Fuel Value Index) by considering several important parameters calorific value, density, ash content and moisture content to characterize the overall quality of the firewood.

To analyze the physical characteristics of the preferred species, FVI was applied using the following method (Bhatt and Badoni 1990).

$\mathrm{FVI}=[$ Calorific value $(\mathrm{KJ} / \mathrm{g}) \times$ Density $(\mathrm{g} / \mathrm{cc})] /[$ Ash content $(\%) \times$ Water content $(\%)]$

Here,

Calorific value $(\mathbf{K J} / \mathbf{g})$ : Samples were dried in a forced air convection oven at $(100 \pm 5)^{\circ} \mathrm{c}$. After that sufficiently drying, part of the sample was ground to pass number 4 sieve ( $4.7 \mathrm{~mm} \mathrm{mesh}$ ), and about $1 \mathrm{~g}$ of ground material was pelleted and burnt in the oxygen bomb calorimeter. Samples stored under controlled conditions $\left(60 \%\right.$ relative humidity, $\left.20^{\circ} \mathrm{c}\right)$ for one month to attain equilibrium moisture content before to measurement. Gross calorific value (gross CV) is the maximum energy available from an oven-dry sample, and net calorific value (net CV) is the energy available from an air-dry sample. Gross CV was measured in an oxygen bomb calorimeter, using the following formula

Gross $\mathrm{CV}=\mathrm{k}\left(\mathrm{t}_{\mathrm{f}}-\mathrm{t}_{\mathrm{i}}\right) / \mathrm{w}$

Where, $\mathrm{K}=$ calorimeter constant in $\mathrm{kcal} / \mathrm{g}$ rescaled to $\mathrm{KJ} / \mathrm{g}$ (i.e multiplied by 4.187), $\mathrm{t}_{\mathrm{f}}-\mathrm{t}_{\mathrm{i}}=$ final and initial temperature of the calorimeter, respectively and $\mathrm{w}=$ sample weight $(1 \mathrm{~g})$

The net $\mathrm{CV}$ was derived using the following formula: Net $\mathrm{CV}=$ gross $\mathrm{CV}$ in $\mathrm{KJ} / \mathrm{g}-$ 4.187(600)(9)(h)

Where, $600=\mathrm{kcal}$ needed to evaporate $1 \mathrm{~L}$ of water, $9=$ stoichiometric reaction constant of hydrogen and oxygen, and $\mathrm{h}=$ mean percent of molecular hydrogen in the sample (6\%) (Montes et al. 2011).

Density (g/cc): Firstly samples were kept in water for 5 days for saturation and diameter of samples was taken using vernier caliper. After saturation of samples, it dried in the oven to take the dry weight. Method of measuring the volume of wood and density are

Volume of wood, $\mathrm{v}=\left(\pi \mathrm{D}^{2} \mathrm{c} / 4\right)$ note $\mathrm{D}=$ average diameter, and $\mathrm{c}=$ length and

Density=DW/SV [DW= dry weight (g), SV= Sample volume] (Ramos et al. 2008).

Ash content (\%): To determine ash content, $2 \mathrm{~g}$ of ground sample was burnt in a muffle furnace at $575 \pm 25^{\circ} \mathrm{C}$ for 15 minutes as per TAPPI standard method, T $211 \mathrm{om}-85$ (Deka et al. 2007).

Water content/ moisture content $(\%)$ : To determine water content, the note of fresh weight of a disc of $2 \mathrm{~cm}$ thickness and $4 \mathrm{~cm}$ diameter of sample was taken and the final weight was taken after drying it in an electric oven at $(100 \pm 5){ }^{\circ} \mathrm{c}$ for $6-8 \mathrm{~h}$ (Bhatt et al. 2004).

Basic statistical parameters such as mean and sum were determined for each tree species. The values of FVI of all the species were arranged according to decreasing order and ranked it. A comparison was made between the ranks of the firewood species obtained on the basis of pair-wise ranking and the ranks calculated from their FVI values. It made a resemblance between two rankings (FVI and pair-wise) of firewood species.

\section{Results and discussion}

\section{Local preferences for firewood species}

A ranking matrix using 12 quality criteria is presented in Tab. 1. Twelve quality criteria such as fast drying, hot flame, ember production, easy flammability, not smoky flame, non -sparking, lightweight during dry, lightweight during wet, bright flame, the difficulty of splitting, long lasting and 
termite resistance were considered for the identification of preferred firewood species. Those criteria were used to identify firewood properties and rank species accordingly by Deka et al. (2007), Abbot et al. (1997) and Abbot and Lowore (1999).

The pair-wise ranking were denoted by the respondents for the most preferred species which were Artocarpus heterophyllus (Kanthal), Swietenia mahagoni (Mehegoni), Mangifera indica (Am), Acacia auriculiformis (Akashmoni), Samanea saman (Rendi), Syzygium fruticosum (Jam), Trema orientalis (Naircha), Albizia lebbek (Kalakoroi), Gmelina arborea (Jogini/Gamar), Elaeocarpus robustus (Jalpai), Aphanamixis polystachya (Roinna/ Pitraj), Aeschynomene aspera (Sola), Diospyros peregrina (Gub), Baccaurea ramiflora (Latkan), Zizyphus jujube (Boroi) etc. Those species were available in the homesteads. It was found that all species had all most desirable firewood characteristics. The availability of firewood derived from the homestead trees of the present study mostly resemblance the same species identified by Miah et al. (2003). The authors identified 26 different tree species in the homesteads of Chittagong district. Miah and Hossain (2002) reported that Artocarpus heterophyllus (Kanthal), Swietenia mahagoni (Mehegoni), Mangifera indica (Am) etc. were homestead dominant species in Narsingdi district. The Authors mentioned that Artocarpus heterophyllus (Kanthal) and Mangifera indica (Am) had multiple uses besides using as fuelwood.

Hassan et al. (2013) reported that highly preferred fuelwood species were Artocarpus heterophyllus (Kanthal) and Mangifera indica (Am), Samanea saman (Rendi), Swietenia mahagoni (Mehegoni), etc. and while less preferred species was Bombax ceiba (Shimul) in Nakla Upazila under Sherpur district. The author showed that local preference depended on the local availability of tree species in homestead forests. Jashimuddin et al. (2006) also mentioned Samanea saman (Rendi), Artocarpus heterophyllus (Kanthal), Mangifera indica (Am), Diospyros peregrina (Gub), Syzygium fruticosum (Jam), Gmelina arborea (Jogini/Gamar), Elaeocarpus robustus (Jalpai), Zizyphus jujube (Boroi) Swietenia mahagoni (Mehegoni), Acacia auriculiformis (Akashmoni) etc. as commonly used firewood species in rural area of Bangladesh.

\section{Ranking of firewood characteristics}

Firewood was characterized based on the factors of fast drying and producing hot flame, easy flammability, the difficulty of splitting, sparking from firewood, weight at the dry condition, ember production, termite resistance, and production of flame and moisture content.

\section{Fast drying and producing a hot flame}

In the study, the respondents mostly identified the quality criteria as a fast drying rate and the ability to produce a hot flame for ranking the preferred species. The study found (Tab. 1) that Artocarpus heterophyllus (Kanthal), Swietenia mahagoni (Mehegoni), Mangifera indica (Am), Acacia auriculiformis (Akashmoni), Samanea saman (Rendi), Trema orientalis (Naircha), Albizia lebbek (Kalakoroi), Aeschynomene aspera (Sola), Gmelina arborea (Jogini/Gamar), Elaeocarpus robustus (Jalpai), and Aphanamixis polystachya (Roinna/ Pitraj), as fast drying species and their rating point were 7.13, 6.57, 6.31, 5.95, 5.71, 5.48, 4.4, 3.08, 3.01, 2.86, 2.22 and 1.92, respectively. Among these species, Artocarpus heterophyllus (Kanthal) were highly preferred species because of having associated with a maximum hot flame. On the other hand, Bombax ceiba (Shimul), Annona muricata (Atafal), Averrhoa carambola (Kamranga) were reported to slow drying rates and produce a flame with low heat.

A study showed that about $62 \%$ households depended on branches of trees for meeting their energy demand (Akther et al. 2010b). Hassan et al. (2013) also mentioned that about 100\% respondents preferred branches as fuel in the different region of Satkhira, Chapai-Nababgang, Sherpur and Cox's Bazar of Bangladesh. The author also noted that Bombax ceiba (Shimul) was less preferred species in the rural area of Bangladesh. Deka et al. (2007) noted that fast drying species produce hot flame during burning and slow drying species produce a flame with low heat. 
Environment, Earth and Ecology Vol. 4 (2020), 15 - 30

DOI: $10.24051 /$ eee/122129

Md. Danesh Miah and Gazi Azizul Islam

Tab. 1. Ranking matrix for 36 firewood species based on 12 quality criteria in rural area of Narsingdi district of Bangladesh.

\begin{tabular}{|c|c|c|c|c|c|c|c|c|c|c|c|c|c|c|c|c|c|c|c|c|c|}
\hline & 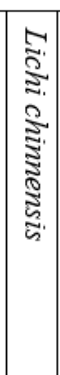 & 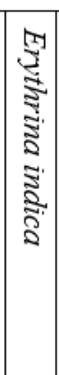 & 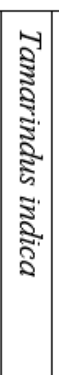 & 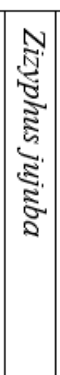 & 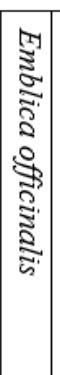 & 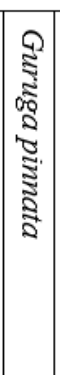 & 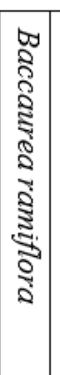 & 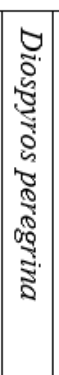 & 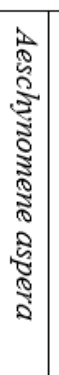 & 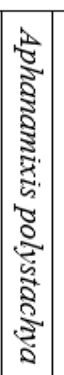 & 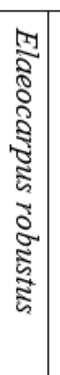 & 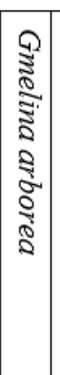 & 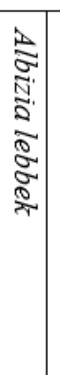 & 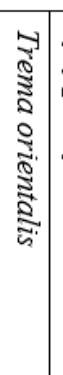 & 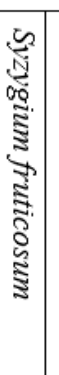 & 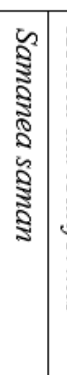 & 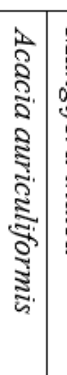 & 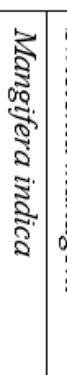 & 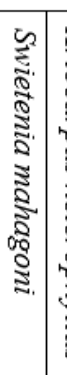 & 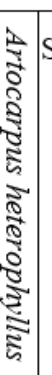 & Scientific Name \\
\hline , & 号 & 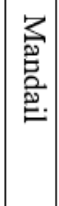 & $\mid \begin{array}{l}-4 \\
\stackrel{0}{\tilde{E}} \\
\text { E }\end{array}$ & \begin{tabular}{|l|} 
\\
0 \\
0 \\
0 \\
0
\end{tabular} & 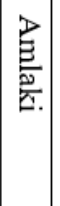 & 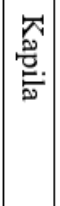 & $\left|\begin{array}{c}t \\
\overrightarrow{2} \\
\vec{T} \\
\hat{E}\end{array}\right|$ & $Q$ & 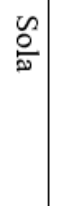 & 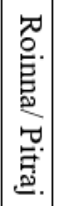 & 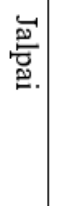 & 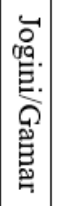 & 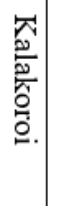 & 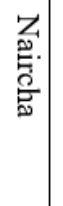 & $\overrightarrow{\widetilde{\hat{E}}}$ & $\begin{array}{l}\pi \\
0 \\
0 \\
2\end{array}$ & 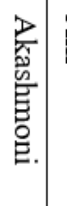 & 当 & 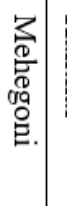 & & Local Name \\
\hline 占 & 品 & $\begin{array}{l}0 \\
\dot{\omega} \\
\end{array}$ & \begin{tabular}{|l|} 
\\
$\dot{+}$ \\
$\infty$
\end{tabular} & $\dot{\hat{\sigma}}$ & $\begin{array}{l}0 \\
\dot{1} \\
\end{array}$ & î & 崫 & \begin{tabular}{l|}
0 \\
$\dot{w}$
\end{tabular} & $\begin{array}{l}\omega \\
\dot{\infty} \\
\dot{\infty}\end{array}$ & \begin{tabular}{|l|} 
\\
\\
\end{tabular} & $\stackrel{N}{N}$ & $\mid \begin{array}{l}n \\
\dot{\alpha} \\
\alpha\end{array}$ & $\stackrel{+}{+}$ & $\begin{array}{c}u \\
1 \\
\infty\end{array}$ & $\begin{array}{l}\omega \\
\dot{0} \\
\dot{0}\end{array}$ & $\begin{array}{ll}u \\
\ddot{v}\end{array}$ & $\begin{array}{l}u \\
0 \\
0 \\
u\end{array}$ & $\dot{\hat{\omega}}$ & is & $\vec{\omega}$ & Fast drying \\
\hline$i_{1}$ & 이 & $\stackrel{0}{\vec{y}}$ & $\mid$\begin{tabular}{l|}
0 \\
$\dot{\infty}$ \\
$\alpha$
\end{tabular} & $\stackrel{0}{\dot{w}}$ & \begin{tabular}{l|}
0 \\
0 \\
$\dot{\omega}$ \\
\end{tabular} & : & 灾 & $\tilde{\omega}$ & $\overrightarrow{6}$ & $\underset{N}{N}$ & $\begin{array}{l}N \\
\ddot{\infty}\end{array}$ & $\begin{array}{c}n \\
\ddot{\alpha}\end{array}$ & $\begin{array}{l}w \\
\stackrel{w}{\sim}\end{array}$ & $\begin{array}{l}\omega \\
\dot{\omega} \\
\dot{\omega}\end{array}$ & $\stackrel{\omega}{\sim}$ & 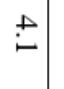 & $\begin{array}{l}u \\
\dot{u} \\
\dot{\omega}\end{array}$ & $\begin{array}{l}u \\
a \\
a\end{array}$ & $\begin{array}{l}u \\
\ddot{y}\end{array}$ & فें & Hot flame \\
\hline $\begin{array}{c}e_{t} \\
\dot{\omega}\end{array}$ & $\stackrel{ }{v}$ & 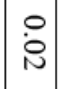 & $\mid$\begin{tabular}{l|}
0 \\
$\infty$ \\
$\infty$ \\
$\infty$
\end{tabular} & $\stackrel{\circ}{\stackrel{0}{ \pm}}$ & : & $\begin{array}{ll}0 \\
ن \\
ن \\
ن\end{array}$ & $\vec{\omega}$ & $\begin{array}{l}1 \\
\stackrel{0}{\circ} \\
\end{array}$ & i & $\begin{array}{l}n \\
\hat{i} \\
\end{array}$ & $\begin{array}{l}N \\
\infty \\
\bullet\end{array}$ & $\mid \begin{array}{l}n \\
0 \\
\omega\end{array}$ & $\stackrel{0}{=}$ & $\stackrel{\circ}{i}$ & $\stackrel{+}{\mathrm{N}}$ & \begin{tabular}{l}
0 \\
$\infty$ \\
$\infty$ \\
\hdashline
\end{tabular} & $\begin{array}{l}u \\
\ddot{\alpha}\end{array}$ & iे & \begin{tabular}{l}
0 \\
0 \\
0 \\
\hdashline
\end{tabular} & $\stackrel{\sim}{\omega}^{\infty} \mathrm{F}$ & Produce ember \\
\hline in & :̊ & $\stackrel{ }{\longrightarrow}$ & $\mid$\begin{tabular}{c|}
0 \\
$\dot{\infty}$ \\
$\infty$
\end{tabular} & 양 & $\mid \begin{array}{l}0 \\
\dot{i} \\
1\end{array}$ & :이 & $\doteqdot$ & \begin{tabular}{l|}
0 \\
$\dot{0}$ \\
$\infty$
\end{tabular} & $\begin{array}{l}\omega \\
\dot{w} \\
w\end{array}$ & 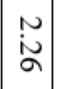 & $\begin{array}{l}N \\
\stackrel{N}{\Delta}\end{array}$ & $\begin{array}{c}n \\
\dot{\sigma}\end{array}$ & : & wa & $\stackrel{N}{y}$ & 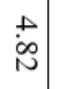 & $\begin{array}{l}u \\
\dot{u} \\
\dot{N}\end{array}$ & $\ddot{a}$ & $\ddot{\omega}$ & 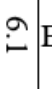 & Easy flammability \\
\hline 分 & $\begin{array}{l}0 \\
\dot{1} \\
\infty\end{array}$ & 唄 & $\mid \begin{array}{l}0 \\
\dot{1} \\
\text { a }\end{array}$ & 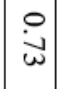 & : & $\begin{array}{l}0 \\
\mathbf{N} \\
\end{array}$ & 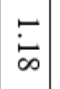 & $\mid \overrightarrow{\omega_{u}}$ & స̃ & $\overrightarrow{\mid \vec{\omega}}$ & $\stackrel{N}{\perp}$ & $\mid \begin{array}{l}n \\
\stackrel{1}{S} \\
\end{array}$ & $\begin{array}{l}N \\
\infty \\
\infty\end{array}$ & 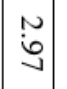 & No & $\begin{array}{l}\omega \\
\vdots \\
=\end{array}$ & $\begin{array}{l}u \\
u \\
:\end{array}$ & $\begin{array}{l}u \\
\ddot{a}\end{array}$ & $\ddot{g}$ & $\stackrel{a}{+} 1$ & Not smoky flame \\
\hline मे & $\dot{9}$ & $\begin{array}{l}0 \\
\dot{ \pm} \\
+\end{array}$ & $\left|\begin{array}{l}0 \\
\dot{\infty} \\
\infty \\
\omega\end{array}\right|$ & $\begin{array}{l}0 \\
\dot{a} \\
\end{array} \mid$ & ìv & $\overrightarrow{u_{1}}$ & $\overrightarrow{\omega_{1}}$ & $\vec{b}$ & $\stackrel{\circ}{\mathbf{N}}$ & $\begin{array}{c}N \\
\stackrel{\omega}{\omega}\end{array}$ & $\begin{array}{l}N \\
\stackrel{\omega}{\omega} \\
\omega\end{array}$ & $\left|\begin{array}{c}n \\
\infty \\
\infty \\
\infty\end{array}\right|$ & $\underset{w}{w} \underset{\omega}{\omega}$ & $\mid \begin{array}{c}N \\
\dot{\omega} \\
\infty\end{array}$ & $\begin{array}{c}\omega \\
\omega \\
\omega\end{array}$ & $\begin{array}{c}\omega \\
\dot{b} \\
\infty\end{array}$ & $\ddot{a}$ & $\dot{\omega}$ & ì山 & $\dot{w}_{\vec{\omega}} 1$ & Non sparking \\
\hline ' & ö & 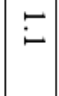 & $\left|\begin{array}{l}0 \\
\dot{u} \\
\alpha\end{array}\right|$ & i & $\stackrel{0}{\underset{\omega}{\omega}}$ & 웅 & $\vec{u}$ & $\begin{array}{c}N \\
\infty \\
\infty\end{array}$ & $\underset{\substack{\infty \\
w}}{+}$ & $\dot{\grave{\alpha}}$ & 志 & $\begin{array}{l}n \\
\dot{N} \\
\end{array}$ & $\stackrel{+}{\dot{\omega}}$ & $\begin{array}{l}u \\
\vdots \\
N\end{array}$ & $\mid$ & $\omega_{\omega}^{u}$ & $\vec{i}$ & $\stackrel{+}{\sim}$ & $\stackrel{+}{*}$ & $\begin{array}{c}w \\
\text { ind } \\
\text { I }\end{array}$ & $\begin{array}{l}\text { Light weight } \\
\text { during dry }\end{array}$ \\
\hline ' & $\stackrel{\circ}{\dot{\omega}}$ & 完 & $\mid$\begin{tabular}{l|}
0 \\
$\dot{i}$ \\
$\omega$
\end{tabular} & $\begin{array}{l}0 \\
\dot{i} \\
\infty\end{array}$ & $\mid \begin{array}{l}0 \\
\dot{i} \\
\infty\end{array}$ & $\begin{array}{l}0 \\
i \\
i \\
N\end{array}$ & $\begin{array}{l}0 \\
0 \\
0\end{array}$ & $\mid \begin{array}{l}0 \\
\dot{j} \\
u \\
u\end{array}$ & $\begin{array}{l}n \\
\text { N }\end{array}$ & 芯 & $\dot{\dot{u}}$ & $\stackrel{v}{\sim}$ & $\begin{array}{l}n \\
a\end{array}$ & 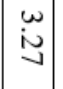 & $\stackrel{\sim}{u}$ & $\begin{array}{l}\omega \\
\vdots \\
\sigma\end{array}$ & $\underset{\sim}{\omega}$ & 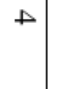 & $\vec{\omega}$ & $\vec{i} \underset{\sim}{\sim} \mid$ & $\begin{array}{l}\text { Light weight } \\
\text { during wet }\end{array}$ \\
\hline$\stackrel{\circ}{\circ}$ & $\stackrel{\circ}{\dot{\omega}}$ & $\stackrel{\circ}{i=}$ & $\begin{array}{l}0 \\
\dot{u} \\
u\end{array}$ & $\begin{array}{l}0 \\
\dot{\alpha}\end{array}$ & $\dot{\omega}$ & $\begin{array}{l}0 \\
\stackrel{ \pm}{ \pm} \\
\end{array}$ & $\begin{array}{l}0 \\
\dot{0} \\
ن\end{array}$ & 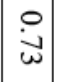 & $\dot{\infty}$ & 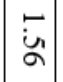 & $\stackrel{\infty}{\infty}$ & 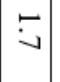 & $\begin{array}{l}n \\
u \\
u\end{array}$ & $\overrightarrow{6}$ & $\underset{\infty}{N}$ & مे & $\begin{array}{l}\omega \\
\dot{\alpha}\end{array}$ & $\tilde{i}_{\infty}$ & $\begin{array}{c}+ \\
\dot{\omega}\end{array}$ & $\dot{\hat{u}}$ & Bright flame \\
\hline बे & $\begin{array}{l}0 \\
\dot{\infty} \\
\infty\end{array}$ & i & 1 & 이 & $\mid$\begin{tabular}{l|}
0 \\
$\infty$ \\
$\infty$ \\
$\infty$
\end{tabular} & 岕 & 5 & $\begin{array}{l}\omega \\
\dot{a} \\
\stackrel{a}{a}\end{array}$ & $\begin{array}{l}0 \\
\dot{0} \\
ن\end{array}$ & $\begin{array}{c}n \\
\dot{a} \\
\grave{2}\end{array}$ & $\begin{array}{l}N \\
\stackrel{N}{ } \\
\dot{u}\end{array}$ & 1 & $\underset{N}{\stackrel{+}{N}}$ & $\stackrel{+}{\stackrel{十}{二}}$ & $\underset{i}{w}$ & $u$ & $\begin{array}{l}u \\
u \\
u\end{array}$ & $\ddot{n}$ & $\begin{array}{l}u \\
\infty\end{array}$ & $\begin{array}{lll}u & \\
\infty & & \\
v & \end{array}$ & $\begin{array}{l}\text { Difficulty of } \\
\text { splitting }\end{array}$ \\
\hline ํ. & 定 & 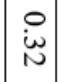 & $\mid \begin{array}{l}0 \\
\infty \\
\omega\end{array}$ & $\stackrel{\circ}{+}$ & $\overrightarrow{\dot{\infty}}$ & $\vec{E}$ & $\dot{i}$ & 孞 & 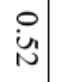 & $\begin{array}{ll}\omega \\
\dot{\partial} \\
u\end{array}$ & Na & 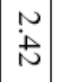 & 畓 & $\dot{\circ}$ & $\begin{array}{c}\omega \\
\dot{\omega} \\
\omega\end{array}$ & $\left|\begin{array}{c}N \\
\omega \\
\omega\end{array}\right|$ & $u_{\omega}$ & $\dot{\dot{\omega}}$ & $\ddot{u}$ & $\begin{array}{c}\infty \\
\underset{\sim}{\sim} \\
\end{array}$ & Long lasting \\
\hline $\begin{array}{l}\stackrel{\circ}{+} \\
\text { a }\end{array}$ & $\stackrel{\circ}{\sim}$ & 吕 & $\mid \begin{array}{l}0 \\
i \\
2\end{array}$ & $\left|\begin{array}{l}0 \\
\dot{u} \\
u\end{array}\right|$ & 灾 & 芯 & $\mid \begin{array}{l}\vec{u} \\
u\end{array}$ & ' & $\stackrel{\circ}{.}$ & $\begin{array}{l}\mid \omega \\
\dot{Q} \\
\alpha\end{array}$ & $\begin{array}{l}N \\
\stackrel{n}{a}\end{array}$ & $\left|\begin{array}{l}n \\
6 \\
j\end{array}\right|$ & $\bar{\omega}$ & \begin{tabular}{|c|}
$\omega$ \\
$\dot{\alpha}$
\end{tabular} & $\stackrel{+}{\dot{\omega}}$ & $\begin{array}{l}0 \\
\dot{0} \\
\dot{0}\end{array}$ & $\dot{\omega}$ & $\begin{array}{l}\omega \\
\dot{\omega} \\
\dot{u}\end{array}$ & $\stackrel{a}{\mathbf{\omega}}$ & $N$ & Termite resistance \\
\hline iे & $\stackrel{0}{\infty}$ & $\hat{i}$ & $\mid \begin{array}{r}\vec{H} \\
+\end{array}$ & $\left|\begin{array}{l}\infty \\
\dot{a} \\
\sigma\end{array}\right|$ & $\mid \begin{array}{l}0 \\
\text { i } \\
\infty\end{array}$ & $\begin{array}{l}F \\
\vec{\omega} \\
\omega\end{array}$ & $\left|\begin{array}{c}+ \\
\dot{*} \\
2\end{array}\right|$ & $\begin{array}{c}\infty \\
\dot{\infty} \\
\infty\end{array}$ & $\begin{array}{l}N \\
\stackrel{N}{\sim}\end{array}$ & $\begin{array}{c}N \\
0 \\
\infty\end{array}$ & $\begin{array}{c}n \\
\stackrel{1}{N}\end{array}$ & $\begin{array}{c}N \\
N \\
\infty\end{array}$ & $\begin{array}{l}w \\
\stackrel{w}{a} \\
\stackrel{N}{N}\end{array}$ & $\left|\begin{array}{c}\omega \\
\stackrel{\omega}{\omega} \\
\infty\end{array}\right|$ & 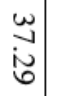 & $\begin{array}{l}\vec{t} \\
\stackrel{u}{n}\end{array}$ & 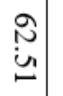 & $\begin{array}{l}a \\
0 \\
\dot{v}\end{array}$ & ڤे & 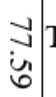 & Total score \\
\hline$\stackrel{N}{\sim}$ & ํ. & 5 & $\infty$ & $\Xi$ & $\bar{a}$ & $\overrightarrow{u_{1}}$ & $\mp$ & $\varpi$ & $\bar{N}$ & $\Xi$ & ธ & 0 & $\infty$ & $v$ & $a$ & $u$ & + & $\omega$ & $N$ & $\mathrm{H}$ & Rank \\
\hline
\end{tabular}


Environment, Earth and Ecology Vol. 4 (2020), 15 - 30

DOI: $10.24051 /$ eee/122129

Md. Danesh Miah and Gazi Azizul Islam

Tab. 1. (cont.) Ranking matrix for 36 firewood species based on 12 quality criteria in rural area of Narsingdi district of Bangladesh.

\begin{tabular}{|c|c|c|c|c|c|c|c|c|c|c|c|c|c|c|c|c|c|c|}
\hline 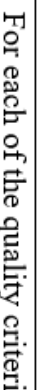 & 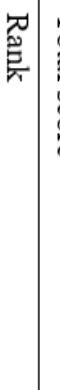 & 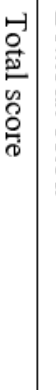 & 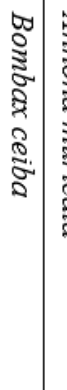 & 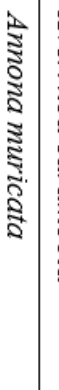 & 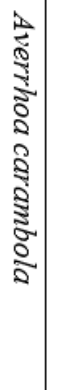 & 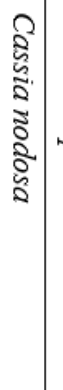 & 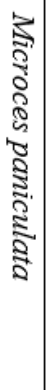 & 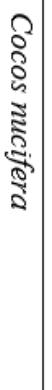 & 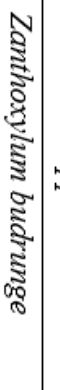 & 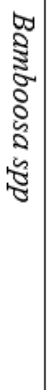 & 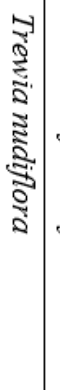 & 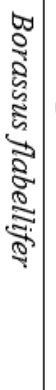 & 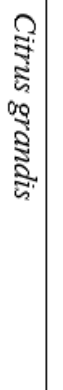 & $\begin{array}{l}2 \\
8 \\
2 \\
0 \\
5 \\
0 \\
\vdots \\
\vdots \\
\vdots \\
0\end{array}$ & 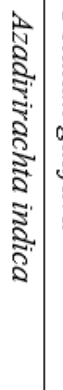 & 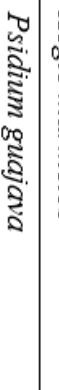 & 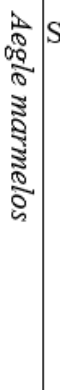 & Scientific Name \\
\hline है & & & 足 & 䔡 & 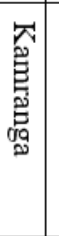 & 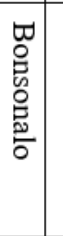 & 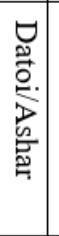 & 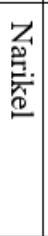 & 悫. & 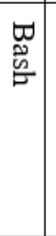 & 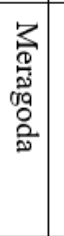 & $\stackrel{\oplus}{\ddot{2}}$ & $\begin{array}{l}\vec{M} \\
\vec{E} \\
\vec{E} \\
\vec{\omega}\end{array}$ & 空 & $\begin{array}{c}Z \\
\mathbb{0} \\
\Xi \\
\mathbb{B}\end{array}$ & 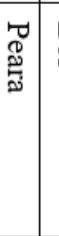 & $\underset{\oplus}{\mathbb{Q}} \mathrm{I}_{\mathrm{I}} \mathrm{L}$ & Local Name \\
\hline 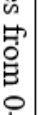 & - & 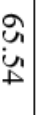 & ڤ̊) & $\stackrel{\circ}{=}$ & $\stackrel{\circ}{\dot{u}_{1}}$ & $\stackrel{\circ}{\sqcup}$ & i & 㘄 & i & : & 品 & $\stackrel{0}{i}$ & $\stackrel{0}{i}$ & $\dot{Q}_{1}$ & 总 & $\begin{array}{l}\circ \\
\pm\end{array}$ & $\underset{\sim}{\stackrel{0}{u}} \mathrm{~F}$ & Fast drying \\
\hline 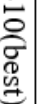 & + & $\begin{array}{l}\omega \\
\infty \\
\dot{\omega} \\
\end{array}$ & $\stackrel{\circ}{\circ}$ & 임 & $\dot{0}_{1}$ & $\stackrel{\circ}{=}$ & $\stackrel{0}{\infty}$ & $\stackrel{\circ}{i}$ & 品 & $\stackrel{\circ}{\stackrel{D}{\omega}}$ & $\stackrel{\circ}{\oplus}$ & i & $\stackrel{\circ}{+}$ & $\begin{array}{l}0 \\
\dot{A} \\
\end{array}$ & $\begin{array}{l}0 \\
\dot{+} \\
\hat{a}\end{array}$ & $\stackrel{\circ}{\oplus}$ & $i_{1} \mathrm{I}$ & Hot flame \\
\hline$\therefore$ & o & & 0 & : & : & $\stackrel{0}{i}$ & : & :일 & is & $\circ$ & i) & : & in & $\dot{i}_{\omega_{1}}$ & i & in & 品 $\mathrm{F}$ & Produce ember \\
\hline 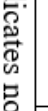 & $n$ & 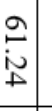 & : & : & $\stackrel{0}{i}$ & : & i & i⿱ & $\stackrel{\circ}{i}$ & $\begin{array}{l}\text { i } \\
\dot{\infty} \\
\infty\end{array}$ & $\begin{array}{l}0 \\
\text { 主 }\end{array}$ & $\begin{array}{l}\circ \\
\ddagger\end{array}$ & 咅 & $\stackrel{\circ}{\perp}$ & $\begin{array}{l}0 \\
\dot{+} \\
\text { a }\end{array}$ & 总 & 임 & Easy flammability \\
\hline $\begin{array}{l}\text { कृ } \\
\text { : }\end{array}$ & o & 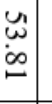 & : & $\stackrel{\circ}{\circ}$ & : & $\stackrel{\circ}{\omega}$ & $\stackrel{0}{\stackrel{0}{\omega}}$ & i & ì & i & $\stackrel{\circ}{\oplus}$ & 䀝 & $\left|\begin{array}{c}0 \\
\dot{1} \\
\infty\end{array}\right|$ & $\begin{array}{l}\text { : } \\
\dot{u}\end{array}$ & 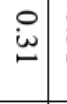 & 㝏 & $\underset{\omega}{\dot{\omega} \omega} 1$ & Not smoky flame \\
\hline 芦 & $u$ & 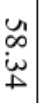 & 임 & : & ' & $\begin{array}{l}0 \\
\dot{U}\end{array}$ & : & : & 이 & 0 & $\begin{array}{l}\circ \\
\doteq\end{array}$ & 定 & 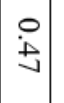 & 宓 & $\begin{array}{c}\stackrel{0}{\omega} \\
\dot{\omega}\end{array}$ & 总 & î̀ & Non sparking \\
\hline $\begin{array}{l}\overline{6} \\
\text { 它 } \\
\\
\end{array}$ & $a$ & $\begin{array}{l}u \\
\vec{v} \\
\vec{v}\end{array}$ & $\dot{\infty}$ & 官 & $\stackrel{0}{\dot{\omega}}$ & $\stackrel{\circ}{=}$ & $\underset{\omega}{\stackrel{0}{\omega}}$ & 总 & $\begin{array}{l} \\
\dot{n}\end{array}$ & $\stackrel{0}{+}$ & $\begin{array}{l} \\
\\
i \\
\infty\end{array}$ & $\begin{array}{l} \\
\text { ü }\end{array}$ & \begin{tabular}{|l|} 
\\
\\
$i$ \\
$i$
\end{tabular} & $i_{1}$ & 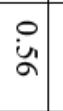 & $\begin{array}{l} \\
\text { i } \\
\text { N }\end{array}$ & $\underset{\sim}{\stackrel{\sim}{N} I}$ & $\begin{array}{l}\text { Light weight } \\
\text { during dry }\end{array}$ \\
\hline 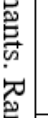 & N & $\underset{\infty}{ \pm}$ & :̊ & 연 & $\stackrel{\circ}{\circ}$ & $\begin{array}{l}0 \\
\stackrel{\infty}{ }\end{array}$ & $\stackrel{0}{\infty}$ & $\dot{S}_{1}$ & $\stackrel{\stackrel{O}{u}}{i}$ & $\underset{\substack{i \\
\text { i }}}{\mid}$ & O & $\underset{\infty}{\substack{\infty \\
\infty}}$ & 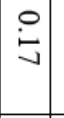 & $\underset{\sim}{\stackrel{0}{N}}$ & $\begin{array}{l}\stackrel{0}{1} \\
\dot{\omega}\end{array}$ & $\stackrel{\circ}{\perp}$ & $\underset{\sim}{\stackrel{i}{N}} \mathrm{I}$ & $\begin{array}{l}\text { Light weight } \\
\text { during wet }\end{array}$ \\
\hline 䈦 & $\Xi$ & $\begin{array}{l}\vec{t} \\
\grave{+} \\
+\end{array}$ & 잉 & 0 & 이 & $\begin{array}{l}0 \\
\infty \\
\infty\end{array}$ & 0 & i & : & $\begin{array}{l}0 \\
\text { i } \\
\text { (n) }\end{array}$ & $\begin{array}{l}0 \\
\vdots \\
\vdots\end{array}$ & $\stackrel{0}{\vdots}$ & $\begin{array}{c}0 \\
\dot{\infty} \\
\infty\end{array} \mid$ & $\begin{array}{l}0 \\
\dot{⿰} \\
\text { a }\end{array}$ & $\stackrel{\circ}{\perp}$ & $\stackrel{\circ}{\omega}$ & $\begin{array}{l}0 \\
\dot{\Delta} \\
\mathbf{H}_{1}\end{array}$ & Bright flame \\
\hline 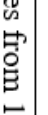 & $\omega$ & 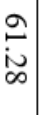 & $\stackrel{0}{=}$ & $\stackrel{\circ}{\circ}$ & $\begin{array}{l}0 \\
\vdots\end{array}$ & $\begin{array}{l}0 \\
\dot{U} \\
n_{1}\end{array}$ & ' & $\stackrel{\circ}{\oplus}$ & $\begin{array}{l}\stackrel{1}{0} \\
\text { i }\end{array}$ & $\begin{array}{l}0 \\
\dot{\sigma}\end{array}$ & $\begin{array}{l}0 \\
\dot{\alpha} \\
\alpha\end{array}$ & $\begin{array}{l}\circ \\
\stackrel{\imath}{\sigma}\end{array}$ & ' & 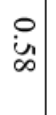 & $\begin{array}{l}\circ \\
\pm\end{array}$ & $\begin{array}{l}\circ \\
\dot{\omega}\end{array}$ & s & $\begin{array}{l}\text { Difficulty of } \\
\text { splitting }\end{array}$ \\
\hline 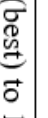 & $\sim$ & $\begin{array}{l}u \\
2 \\
\infty \\
0\end{array}$ & 임 & $\stackrel{0}{\circ}$ & $\stackrel{0}{i}$ & $\begin{array}{l}0 \\
\dot{U} \\
n_{1}\end{array}$ & $\stackrel{0}{i}$ & : & $\begin{array}{l}0 \\
\stackrel{i}{N}\end{array}$ & ○े & $\begin{array}{l}0 \\
\dot{\alpha} \\
\alpha\end{array}$ & $\begin{array}{l}0 \\
\underset{N}{\mathbf{u}}\end{array}$ & $\left|\begin{array}{l}0 \\
u \\
u\end{array}\right|$ & $\stackrel{\circ}{\perp}$ & $\begin{array}{l}\stackrel{0}{1} \\
\dot{\Delta}\end{array}$ & $\begin{array}{l}0 \\
\dot{\omega}\end{array}$ & வे & Long lasting \\
\hline ఫิ & $\infty$ & 感 & $\stackrel{0}{\circ}$ & $\stackrel{0}{\circ}$ & $\stackrel{0}{\dot{0}}$ & $\stackrel{0}{\Xi}$ & $\begin{array}{l}0 \\
\text { i } \\
\text { n }\end{array}$ & 응 & $\begin{array}{l}0 \\
i \\
i\end{array}$ & 品 & $\begin{array}{l}\stackrel{1}{ \pm} \\
\dot{ \pm}\end{array}$ & $\begin{array}{l}0 \\
\text { in }\end{array}$ & :̊̀ & $\begin{array}{l}\stackrel{D}{ \pm} \\
\dot{\omega}\end{array}$ & 客 & ì & 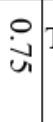 & Termite resistance \\
\hline & & & $\stackrel{\circ}{\ddagger}$ & ڤ̆ & 它 & $\overrightarrow{\omega_{\omega}}$ & $\vec{\infty}$ & $\begin{array}{l}n \\
\sim \\
\sim \\
+\end{array}$ & $\begin{array}{l}n \\
\vdots \\
\omega\end{array}$ & $\begin{array}{l}w \\
i \\
\alpha\end{array}$ & + & $\stackrel{+}{\infty}$ & $\stackrel{+}{\dot{\omega}}$ & $\begin{array}{l}u \\
⿱ 亠 䒑 \\
\end{array}$ & $\begin{array}{l}u \\
\dot{\omega} \\
\dot{\omega}\end{array}$ & $\begin{array}{l}u \\
u \\
\dot{t} \\
t\end{array}$ & $\ddot{\omega}$ & Total score \\
\hline & & & $\omega$ & $\underset{u}{\omega}$ & $\stackrel{w}{\perp}$ & $\underset{\omega}{\omega}$ & $\underset{N}{\sim}$ & $\stackrel{\omega}{\omega}$ & $\tilde{o}$ & กั & $\infty$ & $\mathrm{N}$ & $\tilde{\alpha}$ & $\tilde{u}_{1}$ & $\tilde{A}$ & $\omega$ & & Rank \\
\hline
\end{tabular}




\section{Easy flammability}

The firewood species having easy flammability take less time to start fire and reduce the trouble of initial burning operation. The species (Tab. 1) having easy flammability were Artocarpus heterophyllus (Kanthal), Swietenia mahagoni (Mehegoni), Acacia auriculiformis (Akashmoni), etc., and these species were highly preferred species by local people because the rural people especially women felt comfortable during cooking due to its easy burning properties. Rural people generally women collect branches of trees for cooking as it is already sun-dried. Miah et al. (2003) reported that about $63 \%$ females collect biomass fuel from their home garden. The present study also found branches of Artocarpus heterophyllus (Kanthal), Swietenia mahagoni (Mehegoni), Acacia auriculiformis (Akashmoni) etc, were collected for cooking. Abbot et al. (1997) also reported flammability as a factor that might be expected considering the short drying period.

\section{Difficulty of splitting}

The difficulty of splitting is an important characteristic of firewood in urban areas (Abbot et al. 1997), but in the present study, it was considered as negative criteria. However, it didn't affect the overall ranking. In the rural area, women try to use easy splitting firewood for their convenience of use during cooking. In the present investigation based on the pair-wise comparison, it was found (Tab. 1) that Annona muricata (Atafal), Bombax ceiba (Shimul) and Averrhoa carambola (Kamranga) were easier for splitting compared to the other species. On the other hand, Artocarpus heterophyllus (Kanthal), Swietenia mahagoni (Mehegoni), Mangifera indica (Am), Acacia auriculiformis (Akashmoni), Samanea saman (Rendi), Diospyros peregrine (Gub) were difficult for splitting.

\section{Sparking from firewood}

The tree species having sparking quality (Tab. 1) were Bombax ceiba (Shimul), Microcos paniculata (Datoi/Ashar), Bambusa spp. (Bash), etc. and on the other hand, non-sparking species were Artocarpus heterophyllus (Kanthal), Swietenia mahagoni (Mehegoni), Mangifera indica (Am), Acacia auriculiformis (Akashmoni), Samanea saman (Rendi), etc. Local people favored non-sparking species because sparking from firewood creates hazards to nearby or around the burning places and considered as undesired quality criteria (Deka et al. 2007). The maximum sparking firewood species were less desired to local people for use as fuel for cooking purpose. The present study considered non-sparking quality as positive characteristics.

\section{Weight at the dry condition}

The wood mass or weight was an obvious criterion preferred by rural firewood collectors because usually firewood was head-loaded from source to its point of use (Abbot et al. 1997). The firewood users generally choose the woods which were lightweight during drying that means having the low moisture content of the species. The study found some firewood species (Tab. 1) which had the highest rating, provided by local people for lightweight during dry/having low moisture content criteria. These species were Artocarpus heterophyllus (Kanthal), Swietenia mahagoni (Mehegoni), Mangifera indica (Am), Acacia auriculiformis (Akashmoni), Samanea saman (Rendi), Trema orientalis (Naircha), Albizia lebbek (Kalakoroi), Syzygium fruticosum (Jam), Gmelina arborea (Jogini/Gamar) and Elaeocarpus robustus (Jalpai), and their rating values were 6.22, 6.11, 5.7, 5.58, 5.31, 5.12 , 4.03, 2.87 and 2.62 , respectively. 


\section{Ember production}

The study found that local people preferred species which had the ability to produce ember. Usually, women collect ember after burning firewood for selling to the local tea stall's owner. Long lasting embers have the capacity to produce uniform heat. It was widely used for space heating, brick burning and slow cooking process (Abbot et al. 1997; Deka et al. 2007). From the Tab. 1, it can be seen that among all the species under study, Artocarpus heterophyllus (Kanthal), Swietenia mahagoni (Mehegoni), Mangifera indica (Am), Acacia auriculiformis (Akashmoni), Syzygium fruticosum (Jam), were reported to produce ember and ability to long-lasting burning. The species which have high density can produce better ember (Deka et al. 2007). On the other hand species Bombax ceiba (Shimul), Annona muricata (Atafal), Averrhoa carambola (Kamranga) etc. didn't produce ember but produced a large amount of ash during burning which was less preferred quality by the rural people.

\section{Termite resistance}

Consideration of termite resistance was important criteria for the respondents for storage of collected firewood. In the rural area, most of the people store their firewood for future use and they select the species which have termite resistance. The study found the termite resistance firewood species (Tab. 1) such as Artocarpus heterophyllus (Kanthal), Acacia auriculiformis (Akashmoni), Swietenia mahagoni (Mehegoni) and Syzygium fruticosum (Jam) which had rating values 7.2, 6.31, 6.23 and 4.13, respectively. These species also had a fast drying rate quality. Abbot et al. (1997) also mentioned the quality of termite resistance was important for storing firewood for future use. However, faster drying species were picked up and used sooner. On the other hand, less termite resistance species were Bombax ceiba (Shimul), Annona muricata (Atafal), Averrhoa carambola (Kamranga), Cocos nucifera (Narikel), Erythrina indica (Mandail), etc., because these species had a high moisture content. Purkayastha (1989) reported that high moisture content species were prone to decay and insect attack.

\section{Production of flame}

As firewood characteristics, production of bright flame was not considered as important criteria for selection of firewood because it was not always used for illumination (Abbot et al. 1997; Deka et al. 2007). However, it was found (Tab. 1) that Artocarpus heterophyllus (Kanthal), Mangifera indica (Am), Swietenia mahagoni (Mehegoni), Acacia auriculiformis (Akashmoni), Albizia lebbek (Kala koroi), etc. had bright flame during burning.

\section{Moisture content}

Generally, people didn't use fresh cut firewood in the rural area of Bangladesh. Usually, they collect dry branches of tree species or they keep them for several weeks for drying and then they use it. So, the moisture content of firewood when freshly cut is not an important quality. But according to the respondents (Tab. 1) Bombax ceiba (Shimul), Annona muricata (Atafal), Averrhoa carambola (Kamranga) had a maximum moisture content, compared to other species when freshly cut. The species, Bombax ceiba (Shimul), is light and low-density wood with a coarse texture and straight grain having high moisture content (Purkayastha 1989).

From the ranking matrix (Tab. 1) prepared on the basis of local people's choice, it can be seen that among all the species under study, Artocarpus heterophyllus (Kanthal) was the most preferred firewood species followed by Swietenia mahagoni (Mehegoni), Mangifera indica (Am), Acacia auriculiformis (Akashmoni), Syzygium fruticosum (Jam), Albizia lebbek (Kala koroi), Elaeocarpus robustus (Jalpai), etc. whereas Bombax ceiba (Shimul), Annona muricata (Atafal), Microces paniculata (Datoi/Ashar) were the least preferred ones. Negi (1992) reported that Bombax ceiba (Shimul) was bad fuelwood. 
Environment, Earth and Ecology Vol. 4 (2020), 15 - 30

DOI: $10.24051 /$ eee/122129

Md. Danesh Miah and Gazi Azizul Islam

Tab. 2. Ranking of 36 firewood species using physical properties of firewood in Narsingdi district of Bangladesh.

\begin{tabular}{|c|c|c|c|c|c|c|c|}
\hline 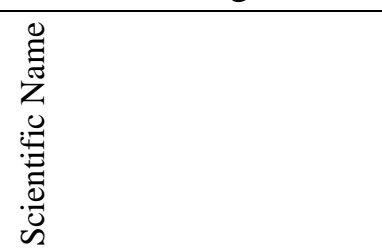 & 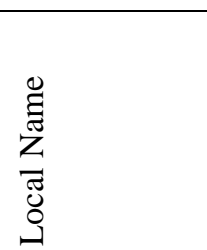 & 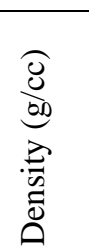 & 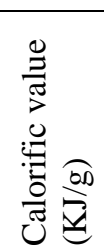 & 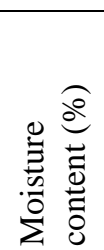 & 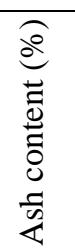 & 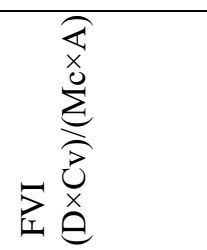 & 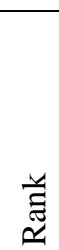 \\
\hline Artocarpus heterophyllus & Kanthal & 0.86 & 20.22 & 43.2 & 1.22 & 3299.408 & 1 \\
\hline Swietenia mahagoni & Mehegoni & 0.82 & 21.86 & 42.8 & 1.28 & 3271.977 & 2 \\
\hline Albizia lebbek & Kalakoroi & 0.81 & 18.79 & 46.29 & 1.21 & 2717.31 & 3 \\
\hline Acacia auriculiformis & Akashmoni & 0.81 & 20.25 & 42.3 & 1.43 & 2711.65 & 4 \\
\hline Syzygium fruticosum & Jam & 0.74 & 20.33 & 43.73 & 1.42 & 2422.709 & 5 \\
\hline Mangifera indica & $\mathrm{Am}$ & 0.72 & 20.72 & 45.2 & 1.56 & 2115.724 & 6 \\
\hline Aegle marmelos & Bel & 0.76 & 17.09 & 47.3 & 1.3 & 2112.278 & 7 \\
\hline Albizia procera & Sil koroi & 0.83 & 18.67 & 50.11 & 1.47 & 2103.684 & 8 \\
\hline Gmelina arborea & Jogini/Gamar & 0.81 & 22.25 & 49.2 & 1.77 & 2069.553 & 9 \\
\hline Elaeocarpus robustus & Jalpai & 0.82 & 20.33 & 45.1 & 1.82 & 2030.969 & 10 \\
\hline Aphanamixis polystachya & Roinna/ Pitraj & 0.8 & 20.41 & 46.31 & 1.75 & 2014.745 & 11 \\
\hline Diospyros peregrina & Gub & 0.69 & 18.52 & 43.78 & 1.68 & 1737.420871 & 12 \\
\hline Zanthoxylum budrunge & Bajna & 0.51 & 17.22 & 44.2 & 1.23 & 1615.384 & 13 \\
\hline Citrus grandis & Jambura & 0.71 & 17.2 & 44.4 & 1.8 & 1528.028 & 14 \\
\hline Azadirirachta indica & Neem & 0.72 & 19.35 & 48.1 & 2.1 & 1379.269 & 15 \\
\hline Emblica officinalis & Amlaki & 0.69 & 19.77 & 48.7 & 2.06 & 1359.751 & 16 \\
\hline Zizyphus jujuba & Boroi & 0.9 & 19.02 & 46.7 & 2.7 & 1357.601 & 17 \\
\hline Tamarindus indica & Tentul & 0.66 & 19.75 & 49.1 & 2.03 & 1307.776 & 18 \\
\hline Baccaurea ramiflora & Latkan & 0.57 & 20.02 & 47.12 & 1.88 & 1288.177 & 19 \\
\hline Lichi chinnensis & Lichu & 0.53 & 17.4 & 59.9 & 1.23 & 1251.679 & 20 \\
\hline Cassia nodosa & Bonsonalo & 0.64 & 19.45 & 50.9 & 1.97 & 1241.41 & 21 \\
\hline Microces paniculata & Datoi/Ashar & 0.45 & 16.73 & 51.44 & 1.85 & 791.1079 & 22 \\
\hline Psidium guajava & Peara & 0.45 & 17.89 & 57.02 & 2.11 & 669.1341 & 23 \\
\hline Cocos nucifera & Narikel & 0.47 & 17.69 & 54.2 & 2.32 & 661.2084 & 24 \\
\hline Cedrella Toona & Rangi & 0.48 & 17.01 & 65.94 & 1.89 & 655.141 & 25 \\
\hline Guruga pinnata & Kapila & 0.54 & 18.22 & 43.00 & 4.7 & 486.82 & 26 \\
\hline Borassus flabellifer & Tal & 0.61 & 15.2 & 49.57 & 3.87 & 483.3297 & 27 \\
\hline Trewia nudiflora & Meragoda & 0.44 & 16.01 & 56.4 & 2.6 & 480.3873 & 28 \\
\hline Samanea saman & Rendi & 0.36 & 14.89 & 60.1 & 3.09 & 288.6451 & 29 \\
\hline Averrhoa carambola & Kamranga & 0.34 & 16.06 & 65.37 & 2.92 & 286.0639 & 30 \\
\hline Bamboosa spp. & Bash & 0.39 & 15.09 & 61.5 & 3.6 & 265.813 & 31 \\
\hline Aeschynomene aspera & Sola & 0.28 & 16.8 & 64 & 3.91 & 187.9795 & 32 \\
\hline Trema orientalis & Naircha & 0.33 & 15.14 & 55.3 & 4.9 & 184.382 & 33 \\
\hline Annona muricata & Atafal & 0.25 & 14.96 & 66.5 & 3.98 & 141.308 & 34 \\
\hline Erythrina indica & Mandail & 0.29 & 15.96 & 56 & 6 & 137.75 & 35 \\
\hline Bombax ceiba & Shimul & 0.24 & 14.42 & 67.65 & 4.02 & 127.2572 & 36 \\
\hline
\end{tabular}


Environment, Earth and Ecology Vol. 4 (2020), 15 - 30

DOI: $10.24051 /$ eee/122129

Md. Danesh Miah and Gazi Azizul Islam

Tab. 3. Comparison on ranking of 36 firewood species on the basis of their Fuel Values and pair-wise quality value.

\begin{tabular}{|c|c|c|c|c|c|}
\hline Scientific Name & Local Name & $\mathrm{FVI}(\mathrm{D} \times \mathrm{Cv}) /(\mathrm{Mc} \times \mathrm{A})$ & Rank & Pair-wise quality value & Rank \\
\hline Acacia auriculiformis & Akashmoni & 2711.65 & 4 & 62.51 & 4 \\
\hline Aegle marmelos & Bel & 2112.278 & 7 & 5.63 & 22 \\
\hline Aeschynomene aspera & Sola & 187.9795 & 32 & 21.21 & 12 \\
\hline Albizia lebbek & Kalakoroi & 2717.31 & 3 & 36.42 & 8 \\
\hline Albizia procera & Sil koroi & 2103.684 & 8 & 6.38 & 21 \\
\hline Annona muricata & Atafal & 141.308 & 34 & 0.66 & 35 \\
\hline Aphanamixis polystachya & Roinna/ Pitraj & 2014.745 & 11 & 25.8 & 11 \\
\hline Artocarpus heterophyllus & Kanthal & 3299.408 & 1 & 77.59 & 1 \\
\hline Averrhoa carambola & Kamranga & 286.0639 & 30 & 1.45 & 34 \\
\hline Azadirirachta indica & Neem & 1379.269 & 15 & 5.39 & 24 \\
\hline Baccaurea ramiflora & Latkan & 1288.177 & 19 & 14.96 & 14 \\
\hline Bamboosa spp. & Bash & 265.813 & 31 & 3.26 & 29 \\
\hline Bombax ceiba & Shimul & 127.2572 & 36 & 0.44 & 36 \\
\hline Borassus flabellifer & Tal & 483.3297 & 27 & 4.18 & 27 \\
\hline Cassia nodosa & Bonsonalo & 1241.41 & 21 & 1.53 & 33 \\
\hline Cedrella Toona & Rangi & 655.141 & 25 & 5.14 & 25 \\
\hline Citrus grandis & Jambura & 1528.028 & 14 & 4.36 & 26 \\
\hline Cocos nucifera & Narikel & 661.2084 & 24 & 2.34 & 31 \\
\hline Diospyros peregrina & Gub & 1737.420 & 12 & 18.18 & 13 \\
\hline Elaeocarpus robustus & Jalpai & 2030.969 & 10 & 26.2 & 10 \\
\hline Emblica officinalis & Amlaki & 1359.751 & 16 & 9.28 & 16 \\
\hline Erythrina indica & Mandail & 137.75 & 35 & 6.97 & 19 \\
\hline Gmelina arborea & Jogini/Gamar & 2069.553 & 9 & 27.8 & 9 \\
\hline Guruga pinnata & Kapila & 486.82 & 26 & 11.35 & 15 \\
\hline Lichi chinnensis & Lichu & 1251.679 & 20 & 6.87 & 20 \\
\hline Mangifera indica & $\mathrm{Am}$ & 2115.724 & 6 & 65.47 & 3 \\
\hline Microces paniculata & Datoi/Ashar & 791.1079 & 22 & 1.98 & 32 \\
\hline Psidium guajava & Peara & 669.1341 & 23 & 5.54 & 23 \\
\hline Samanea saman & Rendi & 288.6451 & 29 & 42.15 & 5 \\
\hline Swietenia mahagoni & Mehegoni & 3271.977 & 2 & 66.03 & 2 \\
\hline Syzygium fruticosum & Jam & 2422.709 & 5 & 37.29 & 6 \\
\hline Tamarindus indica & Tentul & 1307.776 & 18 & 7.74 & 18 \\
\hline Trema orientalis & Naircha & 184.382 & 33 & 36.78 & 7 \\
\hline Trewia nudiflora & Meragoda & 480.3873 & 28 & 4 & 28 \\
\hline Zanthoxylum budrunge & Bajna & 1615.384 & 13 & 2.43 & 30 \\
\hline Zizyphus jujuba & Boroi & 1357.601 & 17 & 8.76 & 17 \\
\hline
\end{tabular}

Note: FVI indicates Fuel Value Index

\section{Physical properties of selected species}

Density, calorific values, moisture content and ash content of selected firewood species along with their fuel value indexes are shown in Tab. 2. The FVI is an important factor for determining the 
suitability of wood as fuel (Abbot et al. 1997; Deka et al. 2007; Kumar et al. 2011; Ramos et al. 2008). In general, high calorific value, high density, low ash, and low moisture content denote an ideal firewood species (Bhatt et al. 2010). The FVI was an alternative ordering procedure of pair-wise ranking to obtain the ranking of firewood species based on physical characteristics of firewood.

In the study, calorific value ranged between 14.42 and $21.86 \mathrm{~kJ} / \mathrm{g}$ in Bombax ceiba (Shimul) and Swietenia mahagoni (Mehegoni), respectively. Wood density varied from 0.24 to $0.86 \mathrm{~g} / \mathrm{cc}$ in Bombax ceiba (Shimul) and Artocarpus heterophyllus (Kanthal). Ash content was the highest 6 (g/g) in Erythrina indica (Mandail) and the lowest $1.21(\mathrm{~g} / \mathrm{g})$ in Albizia lebbek (Kala koroi). The moisture content was the highest, 67.65 (g/g) in Bombax ceiba (Shimul), and the lowest $42.3(\mathrm{~g} / \mathrm{g}$ ) in Acacia auriculiformis (Akashmoni). Shanavas and Kumar (2003) mentioned that high ash content reduced the burning capacity of firewood species.

According to the FVI formula, the highest ranked species was Artocarpus heterophyllus (Kanthal) and its FVI value was 3299.408 followed by Swietenia mahagoni (Mehegoni), Albizia lebbek (Kalakoroi), Acacia auriculiformis (Akashmoni), Syzygium fruticosum (Jam), Mangifera indica (Am), Aegle marmelos (Bel), Albizia procera (Sil koroi), Gmelina arborea (Jogini/Gamar), Elaeocarpus robustus (Jalpai), and Aphanamixis polystachya (Roinna/ Pitraj) and their FVI values were 3271.977, 2717.31, 2711.65 2422.709, 2115.7249, 2112.278, 2103.685, 2069.554, 2030.969 and 2014.745, respectively.

The causes of the highest FVI value were low moisture content and low ash content. Bhatt et al. (2010) and Kumar et al. (2011) mentioned that ideal firewood should have low moisture and low ash content. On the other hand, the lowest ranked species, according to ascending order, were Bombax ceiba (Shimul), Annona muricata (Atafal) and Erythrina indica (Mandail) etc. and where FVI values of them were $127.2573,135.1823$ and 137.75 , respectively. Low density and calorific value negatively affect the FVI value of tree species. Conversely, higher densities of wood improve the FVI values (Deka et al. 2007).

\section{Comparison of local preferences and firewood properties}

The relationship between the ranks obtained from FVI values and pair-wise ranking is shown in Tab. 3. The species having high density and calorific value were quality characteristics of fast drying rate, producing hot flame and ember, the difficulty of splitting and long-lasting burning capacity. The study found that Artocarpus heterophyllus (Kanthal) had the highest density and calorific value of 0.86 $(\mathrm{g} / \mathrm{cc})$ and $20.32(\mathrm{KJ} / \mathrm{g})$, respectively. It also had the highest value of quality criteria such as fast drying rate, producing hot flame and ember, and long-lasting burning capacity was 7.13, 6.96, 8.73, and 8.25, respectively. Deka et al.(2007) also showed that the species Stereospermum chelonoides had the highest density and calorific value of $0.975(\mathrm{~g} / \mathrm{cc})$ and $21.65(\mathrm{KJ} / \mathrm{g})$, respectively and the highest value of quality criteria such as fast drying rate, producing hot flame and ember were 9, 9 and 8, respectively.

The species rank using FVI had the resemblance with the pair-wise ranking such Artocarpus heterophyllus (Kanthal), Swietenia mahagoni (Mehegoni), Acacia auriculiformis (Akashmoni), Aphanamixis polystachya (Roinna/ Pitraj), Bombax ceiba (Shimul), Borassus flabellifer (Tal), Cedrella Toona (Rangi), Elaeocarpus robustus (Jalpai), Emblica officinalis(Amlaki), Gmelina arborea (Jogini/Gamar), Lichi chinnensis (Lichu), and Zizyphus jujube (Boroi), Tamarindus indica (Tentul), Psidium guajava (Peara) and Trewia nudiflora (Meragoda), etc. The coincidence between two ranking procedure means that FVI ranking based on physical properties of firewood corroborates the pair-wise ranking obtained from twelve quality criteria preferred by the local people.

In spite of high FVI values, several species such as Aegle marmelos (Bel), Albizia procera (Sil koroi), Citrus grandis (Jambura), Zanthoxylum budrunge (Bajna) etc. were less preferable to local people. Miah and Hossain (2002) noted that Aegle marmelos (Bel) and Citrus grandis (Jambura) etc. 
were used as fruit species which people didn't use as firewood. On the other hand, Albizia procera (Sil koroi) was less preferred to local people because they were unable to identify some quality characteristics which affect the total ranking and they considered these species produce much ash as a negative characteristic of firewood. The species Zanthoxylum budrunge (Bajna) was not abundant in the study area, and people identified as slow drying rate, producing less ember, less termite resistance and incapable of easy flammability. On the other hand, there were some locally preferred species such as Samanea saman (Rendi), Trema orientalis (Naircha) and Aeschynomene aspera (Sola) which had less FVI values. Local people generally habituated with these species which are locally available and without consideration of high calorific value and density, local preferences varied from place to place (Hassan et al. 2013; Jashimuddin et al. 2006). But the present study considered twelve distinct quality criteria which affected the choice of local people for the selection of firewood species.

The comparison showed that local people's preference for selection of firewood species was based on high heating value, ability to produce ember, fast drying rate, easiness of flammability, the difficulty of splitting and termite resistance.

\section{Conclusion}

The Pair-wise ranking technique identified a number of properties that are used by rural people to determine the desirable firewood. FVI is also an important technique for screening desirable firewood species. The comparison showed that local people prefer firewood species based on high heating value, ability to produce ember, fast drying rate, etc. The higher density of wood enhances the FVI values and in pair-wise ranking, it ensures better quality ember. The results reveal that the ranking order of the firewood species by pair-wise comparison technique used by the rural people has sufficient resemblance with those obtained from FVI values. The study confirms that the supporting physical properties of the firewood species influence the households for their firewood preference for most of the tree species they use for fuels for cooking. The study will support rural energy management practice and energy policy in Bangladesh.

\section{Acknowledgments}

The authors of the study are grateful to Dr. Miki Aturo for supporting this work in his Forest Policy Laboratory of the Faculty of Agriculture, Shinshu University, Nagano, Japan.

\section{References}

Abbot P, Lowore J, Khofi , Werren M (1997) Defining firewood quality: A comparison of quantitative and rapid appraisal techniques to evaluate firewood species from a Southern African Savanna. Biomass and Bioenergy 12( 6): 429-437.

Abbot PG, Lowore JD (1999) Characteristics and management potential of some indigenous firewood species in Malawi. Forest Ecology and Management 119(1-3): 111-121.

Agrawal RK, Singh SP (2001) Energy allocations for cooking in UP households (India): a fuzzy multiobjective analysis. Energy Conversion and Management 42(18): 2139-2154.

Akhter J, Millat-e-Mustafa M, Khan NA, Alam MS (1999) Household biomass fuel energy situation of a forest rich district of Bangladesh. Bangladesh Journal of Agriculture 24(1): 55-65.

Akther S, Miah D, Koike M (2010a) Household adaptations to fuelwood shortage in the old Brahmaputra downstream zone in Bangladesh and implications for homestead forest management. International Journal of Biodiversity Science, Ecosystems Services \& Management 6(3-4): 139-145.

Akther S, Miah MD, Koike M (2010b) Domestic use of biomass fuel in the rural Meghna floodplain areas of Bangladesh. iForest 3: 144-149. 
Akther S, Miah MD, Koike M (2010c) Driving forces for fuelwood choice of households in developing countries: environmental implications for Bangladesh. International Journal of Biodiversity Science \& Management 6(1-2): 35-42.

Balat M, Ayar G (2005) Biomass energy in the world, use of biomass and potential trends. Energy Sources 27(10): 931-940

BBS (2013) District Statistics 2011: Narsingdi.

Bhatt BP, Badoni AK (1990) Characteristics of some mountain firewood shrubs and trees. Energy 15(11): 1069-1070.

Bhatt BP, Sarangi SK, De LC (2010) Fuelwood characteristics of some firewood trees and shrubs of Eastern Himalaya, India. Energy Sources 32(5): 469-474.

Bhatt BP, Todaria NP (1990) Fuelwood characteristics of some mountain trees and shrubs. Biomass 21(3): 233-238.

Bhatt BP, Tomar JMS, Bujarbaruah KM (2004) Characteristics of some firewood trees and shrubs of the North Eastern Himalayan region, India. Renewable Energy 29(8): 1401-1405.

Bungart R, Huttl RF (2001) Production of biomass for energy in post-mining landscapes and nutrient dynamics. Biomass and Bioenergy 20(3): 181-187.

Chowdhury MSH, Koike M, Akther S, Miah D (2011) Biomass fuel use, burning technique and reasons for the denial of improved cooking stoves by forest user groups of Rema-Kalenga wildlife sanctuary, Bangladesh. International Journal of Sustainable Development and World Ecology 18(1): 88-97.

Cuvilas C, Lhate I, Jirjis R, Terziev N (2014) The characterization of wood species from Mozambique as a fuel. Energy Sources 36(8): 851-857.

Deka D, Saikia P, Konwer D (2007) Ranking of fuelwood species by fuel value index. Energy Sources Part A, 29(16): 1499-1506.

Demirbas A (2004) The importance of biomass. Energy Sources 26(4): 361-366.

Eusuf M (1997) Dissemination of Biogas Technology in Bangladesh. National Seminar on Renewable Energy for Poverty Alleviation (NSREPA-97), Institute of Engineers, Bangladesh.

FAO (1997) Biomass Energy in ASEAN Member Countries. Bangkok.

Fuwape JA, Akindele SO (1997) Biomass yield and energy value of some fast-growing multipurpose trees in Nigeria. Biomass and Bioenergy 12(2): 101-106.

Hassan MK, Halder P, Pelkonen P, Pappinen A (2013) Rural households' preferences and attitudes towards biomass fuels - results from a comprehensive field survey in Bangladesh. Energy, Sustainability and Society 3(24): 1-14.

Huda ASN, Mekhilef S, Ahsan A (2014) Biomass energy in Bangladesh: Current status and prospects. Renewable and Sustainable Energy Reviews 30: 504-517.

Jain RK, Sing B (1999) Fuelwood characteristics of selected indigenous tree species from central India. Bioresource Technology 68(3): 305-308.

Jashimuddin M, Masum KM, Salam MA (2006) Preference and consumption pattern of biomass fuel in some disregarded villages of Bangladesh. Biomass and Bioenergy 30(5): 446-451.

Kataki R, Konwer D (2002) Fuelwood characteristics of indigenous tree species of north-east India. Biomass and Bioenergy 22(6): 433-437.

Kumar JIN, Patel K, Kumar RN, Bhoi RK (2011) An evaluation of fuelwood properties of some aravally mountain tree and shrub species of Western India. Biomass and Bioenergy 35(1): 411-414.

Lucia LA, Argyropoulos DS, Adamopoulos L, Gaspar AR (2006) Chemicals and energy from biomass. Canadian Journal of Chemistry 84(7): 960-970.

MacDonald DH, Adamowicz WL, Luckert MK (2001) Fuelwood collection in North-Eastern Zimbabwe: valuation and caloric expenditures. Journal of Forest Economy 7(1): 29-51.

McKendry P (2002) Energy production from biomass (part 1): overview of biomass. Bioresource Technology 83(1): 37-46.

Miah MD, Ahmed R, Uddin MB (2003) Biomass fuel use by the rural households in Chittagong region, Bangladesh. Biomass and Bioenergy 24(4-5): 277-283.

Miah MD, Hossain MK (2002) Tree resources in the floodplain areas of Bangladesh. Schweiz. Z. Forstwes 153(10): 385-391.

Miah MD, Kabir RRMS, Koike M, Akther S, Shin MY (2010) Rural household energy consumption pattern in the disregarded villages of Bangladesh. Energy Policy 38(2): 997-1003. 
Miah MD, Koike M, Shin MY, Akther S (2011) Forest biomass and bioenergy production and the role of CDM in Bangladesh. New Forest 42(1): 63-84.

Mitchual SJ, Frimpong-Mensah K, Darkwa NA (2014) Evaluation of fuel properties of six tropical hardwood timber species for briquettes. Journal of Sustainable Bioenergy Systems 4: 1-9.

Montes CS, Silva DAD, Garcia RA, Muniz GIBD, Weber JC (2011) Calorific value of Prosopis african and Balanites aesgyptiaca wood: Relationships with tree growth, wood density and rainfall gradients in the West African Sahel. Biomass and Bioenergy 35(1): 346-353.

Mukul SA, Tito MR, Munim SA (2014) Can homegardens help save forests in Bangladesh? Domestic biomass fuel consumption patterns and implications for forest conservation in south-central Bangladesh. International Journal of Research on Land-use Sustainability 1: 18-25.

Negi SS (1992) Text book of forest utilization (Wood and non-wood forest products). Bishen Singh Mahendra Pal Singh, Dehra Dun, India.

Pachauri S (2004) An analysis of cross-sectional variations in total household energy requirements in India using micro survey data. EnergyPolicy 32(15): 1723-1735.

Puri S, Singh S, Bhushan B (1994) Evaluation of fuelwood quality of indigenous and exotic tree species of India's semiarid region. Agroforestry Systems 26(2): 123-130.

Purkayastha SK (1989) Common commecial timbers and wood based panel products of India. Bishen Singh Mahendra Pal Singh, Dehradunn, India.

Purohit AN, Nautiyal AR (1987) Fuel wood value index of Indian mountain tree species. The International Tree Crops Journal 4(2-3): 177-182.

Ramos MA, Muniz de Medeiros P, Santos de Almeida AL, Feliciano ALP, Paulino de Albuquerque U (2008) Can wood quality justify local preferences for firewood in an area of caatinga (dryland) vegetation? Biomass and Bioenergy 32(6): 503-509.

Rao MN, Reddy BS (2007) Variations in energy use by Indian households: an analysis of micro level data. Energy 32(2): 143-153.

Sarker MAR, Islam SMN (1998) Rural energy and its utilization in Bangladesh. Energy 23(9): 785789.

Shanavas A, Kumar BM (2003) Fuelwood characteristics of tree species in homegardens of Kerala, India. Agroforestry Systems 58(1): 11-24.

Singh BP, Panigrahi MR, Ray HS (2000) Review of biomass as a source of energy for India. Energy Sources 22(7): 649-658.

Werther J, Saenger M, Hartge EU, Ogada T, Siagi Z (2000) Combustion of agricultural residues. Progress in Energy and Combustion Science 26(1): 1-27.

Wijayatunga PDC Attalage RA (2002) Analysis of household cooking energy demand and its environmental impact in Sri Lanka. Energy Conversion and Management 43(16): 2213-2223.

Xiaohua W, Zhenming F (1996) Survey of rural household energy consumption in China. Energy 21(78): 703-705. 